\title{
BMJ Open Nurses' sleep quality, work environment and quality of care in the Spanish National Health System: observational study among different shifts
}

\author{
Teresa Gómez-García, ${ }^{1}$ María Ruzafa-Martínez, ${ }^{2}$ Carmen Fuentelsaz-Gallego, ${ }^{3}$ \\ Juan Antonio Madrid, ${ }^{4}$ Maria Angeles Rol, ${ }^{4}$ María José Martínez-Madrid, ${ }^{5}$ \\ Teresa Moreno-Casbas, ${ }^{1}$ on behalf of the SYCE and RETICEF Group
}

\begin{abstract}
To cite: Gómez-García T, Ruzafa-Martínez M, Fuentelsaz-Gallego C, et al. Nurses' sleep quality, work environment and quality of care in the Spanish National Health System: observational study among different shifts. BMJ Open 2016;6: 012073. doi:10.1136/bmjopen-2016012073
\end{abstract}

- Prepublication history for this paper is available online. To view these files please visit the journal online (http://dx.doi.org/10.1136/ bmjopen-2016-012073).

Received 29 March 2016 Revised 31 May 2016 Accepted 1 June 2016

CrossMark

For numbered affiliations see end of article.

Correspondence to Dr T Moreno-Casbas; mmoreno@isciii.es

\section{ABSTRACT}

Objective: The main objective of this study was to determine the relationship between the characteristics of nurses' work environments in hospitals in the Spanish National Health System (SNHS) with nurse reported quality of care, and how care was provided by using different shifts schemes. The study also examined the relationship between job satisfaction, burnout, sleep quality and daytime drowsiness of nurses and shift work.

Methods: This was a multicentre, observational, descriptive, cross-sectional study, centred on a selfadministered questionnaire. The study was conducted in seven SNHS hospitals of different sizes. We recruited 635 registered nurses who worked on day, night and rotational shifts on surgical, medical and critical care units. Their average age was 41.1 years, their average work experience was 16.4 years and $90 \%$ worked full time. A descriptive and bivariate analysis was carried out to study the relationship between work environment, quality and safety care, and sleep quality of nurses working different shift patterns.

Results: $65.4 \%$ (410) of nurses worked on a rotating shift. The Practice Environment Scale of the Nursing Work Index classification ranked 20\% (95) as favourable, showing differences in nurse manager ability, leadership and support between shifts ( $p=0.003) .46 .6 \%$ (286) were sure that patients could manage their self-care after discharge, but there were differences between shifts $(\mathrm{p}=0.035)$. 33.1\% (201) agreed with information being lost in the shift change, showing differences between shifts $(p=0.002)$. The Pittsburgh Sleep Quality Index reflected an average of 6.8 (SD 3.39), with differences between shifts ( $p=0.017)$.

Conclusions: Nursing requires shift work, and the results showed that the rotating shift was the most common. Rotating shift nurses reported worse perception in organisational and work environmental factors. Rotating and night shift nurses were less confident about patients' competence of self-care after discharge. The most common nursing care omissions reported were related to nursing care plans. For the Global Sleep Quality score, difference were found between day and night shift workers.

\section{Strengths and limitations of this study}

- This is one of the first studies designed primarily to investigate shift work and the relationships with nurse organisational factors and nurse reported quality of care.

- 635 nurses from seven Spanish hospitals took part in the study, representing hospitals of different sizes (small, medium and large) and different specialties (surgical, medical and critical care).

- The cross sectional design limited our ability to infer causal relationships between the characteristics of the nurses' work environment, nurse reported quality of care and the provision of care through different shifts schemes.

- The survey did not include some aspects of shift work, including overtime, breaks during shifts and total hours worked per week, implying that some unmeasured factors may not have been included.

- We were not able to include any information about nurses' work-life balance or about the proportion of nurses with family commitments.

\section{INTRODUCTION}

International health agencies and nursing associations are aware that unsafe and unhealthy work conditions affect the quality of service delivery and employee health, productivity and retention. The International Council of Nurses noted that establishing positive practice environments across worldwide health sectors is of paramount importance if patient safety and the well being of health workers are to be guaranteed. ${ }^{1}$ Furthermore, one of the four priority action areas that the WHO Regional Office for Europe has identified in its technical guide 'The European strategic directions for strengthening nursing and midwifery towards health 2020 goals' is to promote a positive work environment. The guide also points out that healthy workplace practice 
needs to be monitored and evaluated so that information is available to continuously improve working conditions through research and development. ${ }^{2}$

The number of nursing research studies is increasing, showing that 'the nursing research carried out makes a marked difference to frontline care delivery'. ${ }^{3}$ A substantial part of the literature, largely from North America but increasingly from other countries, has shown that hospitals with consistently positive work environments had lower nurse burnout and turnover rates, and that nurses had less intention to leave their current position and were likely to be less dissatisfied with their jobs. ${ }^{4-8}$ Better work environments have also been linked to the overall quality of care and nursing care provided to patients. Several studies have shown that in hospitals with more favourable environments, there were fewer nurses who thought that the quality of care on their unit was fair or poor, more nurses reported that their patients were ready for discharge ${ }^{46}$ and fewer nurses reported leaving nursing care tasks undone. ${ }^{9}$

Furthermore, positive work environments have been associated with nurse sensitive patient outcomes. Recent studies have found that better nurse work environments are associated with lower hospital acquired pressure ulcers, ${ }^{10} 30$ day readmissions in Medicare patients undergoing surgery, ${ }^{11}$ and 30 day surgical mortality and failure to rescue. ${ }^{12}$ Likewise, patients in hospitals gave the hospitals a higher overall rating if they had a better nurse work environment, and were more likely to recommend the hospital and reported more positive care experiences with nurse communication. ${ }^{13}$

The work environment conceptual framework includes work organisation and the organisational culture, as well as the attitudes, values, beliefs and practices that are demonstrated on a daily basis in the organisation and which affect the mental and physical well being of the employees. Extensive research has identified nine psychosocial factors that pose the greatest risk to workers' health: job content, workload and work pace, work schedule, control, environment and equipment, organisational culture and function, interpersonal relationships at work, role in the organisation, and the home and work interface. $^{14}$

Many of these psychosocial factors have been studied in nurse practice environments across different countries and several differences have been found. ${ }^{15-17}$ The Spanish RN4Cast study showed that $50 \%$ of nurses were dissatisfied with their work schedule, which was higher than in 11 other European countries. ${ }^{18}$ Their work schedule included shift work, night shifts, inflexible schedules, unpredictable hours and long or unsociable hours. ${ }^{14}$ There is an increasing trend towards studying the consequences of long shifts on patient and nurse outcomes, ${ }^{19} 20$ but less attention has been paid to the impact of shift work on nurses' outcomes, even though shifts are a common working pattern for nursing staff. Nursing staff who work shifts tend to experience problems in four main areas, caused by the desynchronisation of the endogenous physiological system of circadian rhythms: ${ }^{21}$ increased fatigue and sleepiness caused by a decreased amount of sleep; poorer general physiological and psychological health; family and social life issues; the quality of the work itself; and the satisfaction they derive from it. ${ }^{22}$

Several studies have analysed shift changes, night working and the resulting sleep disorders, as a risk factor for nurses' health and for patient safety. A review ${ }^{23}$ suggested that fatigue caused by rotating shifts may negatively affect the health of nurses and reduce efficiency, safety and patient care. There was a broad consensus on the negative effects of rotating night shifts and the impact on patient safety, patient conditions, medication errors, patient problem management and child mortality, with a greater impact on nurses over 40 years of age.

Furthermore, recently published studies ${ }^{19} 24$ have shown that working shifts has a strong influence on nurses' job satisfaction, burnout, ${ }^{25}$ intention to leave the hospital or even the profession. Wisetborisut et $a l^{26}$ found that the prevalence of burnout in shift workers was $25 \%$ compared with $15 \%$ in non-shift workers, and having more sleeping hours per day was associated with a lower odds of burnout among shift workers. Nurses working shifts, including night shifts, are subject to a cumulative sleep debt, a decreased quantity and quality of sleep, and continuous sleep deprivation. ${ }^{23}$ They are vulnerable to work related fatigue and, consequently, experience excessive daytime sleepiness. ${ }^{27}$

The majority of the available evidence regarding shift work has focused on nurses' health and sleep problems and experience, or work-life balance. Fewer studies have addressed nurses' perceived experience of care and the work environment, although sleep deprivation also leads to irritability, bad moods, reduced communication skills and ability to cope with the emotional demands of the workplace. ${ }^{28}$ In addition, it produces personality changes and difficulty with personal relationships, ${ }^{29}$ and could impair a nurse's ability to respond to patient care needs. ${ }^{30}$

Therefore, the main objective of this study was to determine the relationship between the characteristics of the nurses' work environments in hospitals in the Spanish National Health System (SNHS) with nurse reported quality of care and how care was provided using different shifts schemes. The study also examined the relation between job satisfaction, burnout, sleep quality and daytime drowsiness of nurses and shift work.

\section{METHODS \\ Design}

A multicentre, observational, descriptive, cross sectional study was conducted in seven SNHS hospitals that were involved in a previous study with European funding (RN4CAST) and expressed their interest in the study. Baseline data were provided by the Hospital Universitario Vall d'Hebron (Barcelona), Complejo Hospitalario 
Universitario de A Coruña (A Coruña), Hospital Universitario de Fuenlabrada (Madrid), Hospital Universitario Virgen de la Arrixaca (Murcia), Complejo Asistencial de Palencia (Palencia), Hospital Doctor José Molina Orosa (Canarias) and Hospital del Mar (Barcelona).

The hospitals were classified according to the number of patient beds available: small hospitals had $<199$ beds, medium hospitals had 200-499 beds and large hospitals had $>500$ beds. We included three types of hospital units in the study: medical, surgical and critical care units. All registered nurses working in the selected hospitals were included in the study if they were providing direct patient care in medical, surgical or critical care units during the study period.

\section{Sampling}

A multistage stratified sampling for nurses' participant selection was conducted. Stratified sampling by hospital size was conducted among all participants in the European RN4CAST project, ${ }^{15}$ carried out between 2008 and 2011, and this identified two major, two medium and three small hospitals. We then carried out a stratified sampling by type of unit-medical, surgical or intensive care-and the nurses working in those units were invited to participate. Data were collected between September 2012 and December 2014.

\section{Measures}

A self-administered questionnaire was developed and used to collect different variables from the nurses:

- Demographic and education measures, including variables such as gender, age, education level, position and department.

- Self-reported labour and shift work measures, type of employment (full time or part time) and years of experience. Shift work is presented as day shifts, including fixed morning and afternoon and 12 hour fixed days; night shifts, including fixed night and 12 hour night shifts; and rotating shifts, including combinations of morning, afternoon and nights shifts and anti-stress shifts.

- Nurse staffing was calculated as the mean number of nurses working in the unit on the last shift before they completed the questionnaire.

- The patient to nurse ratio calculated based on patients assigned to nurses on their last shift.

- Self-reported assessment measures of nursing professionals about the safety and quality of care provided to the patient were evaluated by seven questions. Three questions evaluated quality and safety with four possible options, ranging from bad to excellent; two measured the assurance of quality of care and patient safety, ranging from not sure to very sure; one measured agreement of seven aspects of workers safety, on a Likert scale, ranging from totally disagree to totally agree; and one measured the frequency of adverse events, on a Likert scale, ranging from never to every day.

- Job satisfaction with current work was rated on a 5 point scale as very dissatisfied (1), somewhat dissatisfied (2), fair (3), somewhat satisfied (4) and very satisfied (5). We also assessed satisfaction with their professional status, autonomy, flexibility schedule, salary, continuous learning opportunities and holidays, and if they were satisfied with their choice of nursing as a profession, on the 5 point scale described before.

- Organisation of provision of care, measured by nursing tasks that had not been completed because of lack time, and by non-nursing tasks performed more frequently. Those items were used in the RN4CAST study. ${ }^{31}$

To measure the rest of the variables, we used the following validated tools:

- Practice Environment Scale of the Nursing Work Index (PES-NWI), Spanish validated version. ${ }^{32}$ This measure consists of five subscales rated on a 4 point scale, with responses ranging from strongly disagree to strongly agree: 'collegial nurse-physician relations', 'nurse participation in hospital affairs', 'nursing foundations for quality of care', 'nurse manager ability, leadership and support of nurses', and 'staffing and resource adequacy'.

- The Maslach Burnout Inventory (MBI) adapted for the Spanish population. ${ }^{33}$ The Maslach's manual ${ }^{34}$ provides different cut-off points to establish the dimensions categories in relation to the study scope -in our case, the medicine area. The dimensions were classified into low, medium and high burnout, according to the following scores: emotional exhaustion (low $\leq 18$, medium 19-26, high $\geq 27$ ); personal accomplishment (low $\geq 40$, medium 39-34, high $\leq 33$ ); and depersonalisation (low $\leq 5$, medium 6-9, high $\geq 10$ ). A high burnout score was when two or three dimensions had high levels; medium when two or three had medium levels or there was one dimension in each level; and low when two or three had low levels. ${ }^{35}$

Finally, we used these three instruments to record the sleep nurses' profile, daytime sleepiness and sleep quality:

- Horne and Östberg Morningness-Eveningness Scale. ${ }^{36}$ This scale comprises 19 questions, with values ranging from 19 to 86 . Evening types score up to 41, intermediate types score $42-58$ and morning types exceed 59. This scale also has an abbreviated version of five questions, providing values between 4 and 25, with up to 11 classified as an evening type, 12-17 being intermediate and 18 or more being morning type.

- Epworth Scale. ${ }^{37}$ This comprises eight questions with four possible answers from "would never doze or fall asleep" to "high chance of dozing or falling asleep". The scale ranges from 0 to 24 points, and higher 
scores indicate higher daytime sleepiness: low or nonexistent up to 6 points, a middle level of drowsiness between 7 and 8 and excessive sleepiness if the score is $>9$.

- Pittsburgh Sleep Quality Index (PSQI). ${ }^{38}$ The PSQI has 19 questions with seven areas of measurement: subjective sleep quality, sleep latency, sleep duration, habitual sleep efficiency, sleep disturbances, use of sleeping medication and daytime dysfunction. Each area ranges between 0 and 3 points, with higher scores reflecting greater difficulty. The combined score ranges from 0 (easy sleep) to 21 points (severe difficulty).

\section{Ethical considerations}

The project was approved by the Spanish Health Research Fund (Fondo de Investigaciones Sanitarias PI11/00646). All participants were volunteers, who provided written informed consent and could have withdrawn from the study at any time. Confidentiality was guaranteed. Participants were assigned an identifying code number that was maintained throughout the research documents and data. The proposal was evaluated by a peer review process and was approved by the Spanish Research Ethics Committee. ${ }^{39}$

\section{Analysis}

A descriptive analysis was conducted, using relative and absolute frequency measures, for qualitative variables and mean and SD measures for quantitative variables. An analysis of variance was conducted through Scheffe's and Bonferroni's multiple comparison tests, taking $95 \%$ as the level of confidence, in order to study differences in different quantitative variables in different shifts. A $\chi^{2}$ analysis (95\% level of confidence) was conducted to study the relation between qualitative variables in different shifts. All data were analysed with IBM SPSS Statistics, V.22.0.

\section{RESULTS}

\section{Hospital and nurse characteristics}

Seven hospitals participated in the study: three small, two medium and two large. Of the 115 hospital units who took part, $40 \%$ were surgical care units, $15 \%$ were critical care units and $45 \%$ were medical care units. The sample comprised 635 nurses, $87.2 \%$ (551) women, with an average age of 41.1 years (SD 10.03 years). All nurses had a bachelor degree, $19.2 \%$ (122) also had a nursing specialty or a master's degree, $3.9 \%$ (25) had an Advance Studies Degree and 0.5\% (3) had a PhD. Their average work experience was 16.4 years (SD 9.38); $90 \%$ (558) of nurses worked full time and 28\% (169) had completed 51-120 hours of continuing education in the past 24 months.

Just under two-thirds $(65.4 \%, 410)$ worked on a rotating shift, $23.3 \%$ (146) worked on a day shift and $11.3 \%$ (71) worked on a night shift. The average number of hours worked per day was 9.1 (SD 2.51), with a ratio of 8 patients to 1 nurse (SD 5.25) ratio. The day shift ratio was 6.4 (SD 3.26), the night shift was 8.6 (SD 4.9) and the rotating shift ratio was 8.5 (SD 5.7); these differences were significant $(p<0.001)$, particularly between the day shift and night shift $(\mathrm{p}=0.017)$, and the day shift and the rotating shift $(\mathrm{p}<0.001)$.

Work environment, quality and safety of nursing care, and organisation of provision of care

According to the PES-NWI, 39\% of nurses (186) worked in unfavourable hospitals, $41 \%$ (195) in mixed hospitals and $20 \%$ (95) in favourable hospitals. Higher scores were found for the factors 'nursing foundations for quality care' and 'nurse manager ability, leadership and support', with scores of 2.58 (SD 0.55) and 2.54 (SD 0.71 ), respectively, on a scale of 1 (worst score) to 4 (best score). There was a significant difference in the scores for the latter category depending on the shift ( $p=0.003)$, with day staff reporting a better work environment than night staff ( $p=0.005)$ (table 1$)$. 'Nurse participation in hospital affairs' had the lowest score (mean 2.05 (SD 0.52)).

\section{Quality and safety of nursing care}

The quality of nursing care was rated as good or excellent by $83.7 \%$ (519) of nurses. In addition, $46.6 \%$ (286) were very sure or sure that the patients could manage their self-care after discharge, but there were differences between shifts $(p=0.035)$, with $57.3 \%$ (82) of the day shift staff being very sure or sure compared with $42.6 \%$

Table 1 Scores for the Practice Environment Scale of the Nursing Work Index (mean and differences)

\begin{tabular}{|c|c|c|c|c|c|}
\hline \multirow[b]{2}{*}{ PES-NWI factor } & \multirow[b]{2}{*}{$\begin{array}{l}\text { Global score } \\
\text { (mean (SD)) }\end{array}$} & \multicolumn{4}{|c|}{ Actual shift work } \\
\hline & & $\begin{array}{l}\text { Day } \\
\text { (mean (SD)) }\end{array}$ & $\begin{array}{l}\text { Rotating } \\
\text { (mean (SD)) }\end{array}$ & $\begin{array}{l}\text { Night } \\
\text { (mean (SD)) }\end{array}$ & $\begin{array}{l}\text { ANOVA } \\
\text { F ( } p \text { value) }\end{array}$ \\
\hline Staffing and resource adequacy & $2.53(0.689)$ & $2.55(0.685)$ & $2.54(0.680)$ & $2.41(0.755)$ & $1.15(0.319)$ \\
\hline Collegial nurse-doctor relations & $2.49(0.663)$ & $2.52(0.707)$ & $2.48(0.642)$ & $2.47(0.709)$ & $0.15(0.861)$ \\
\hline Nurse manager ability, leadership and support & $2.54(0.714)$ & $2.69(0.768)$ & $2.52(0.690)$ & $2.34(0.692)$ & $5.73(0.003)$ \\
\hline Nursing foundations for quality care & $2.58(0.547)$ & $2.65(0.562)$ & $2.53(0.534)$ & $2.66(0.571)$ & $2.99(0.051)$ \\
\hline Nurse participation in hospital affairs & $2.05(0.519)$ & $2.09(0.630)$ & $2.04(0.477)$ & $2(0.505)$ & $0.72(0.487)$ \\
\hline
\end{tabular}


(29) of the night staff and $43.6 \%$ (174) of the rotating shift. Also, $46.6 \%$ (283) were somewhat confident that the hospital management would act to solve any problems of quality and safety they had reported regarding patient care but there were differences $(p=0.025)$ between the day staff $(70.4 \%, 100)$, night staff $(64.2 \%$, $43)$ and rotating staff $(64.1 \%, 253)$, who gave this answer. We found that $36.4 \%$ (225) of nurses felt that the quality of patient care had deteriorated during the past year and $62.7 \%$ (388) said that patient safety was good or excellent with no differences between shifts to both questions.

With regard to overall hospital safety, $42 \%$ (256) agreed or strongly agreed that errors were used against them and $33.1 \%$ (201) agreed or strongly agreed that information was lost during shift changes. Night staff were more likely to agree or strongly agree $(54.4 \%, 37)$ than rotating $(42.5 \%, 170, \mathrm{p}=0.023)$ or day $(33.8 \%, 47$, $\mathrm{p}=0.002$ ) staff. Also, $58.4 \%$ (356) did not feel free to question the decisions or actions of their superiors while $72.3 \%$ (442) agreed or strongly agreed that the unit went out of its way to ensure that errors were not repeated in the future. We report that $52.9 \%$ (322) agreed or strongly agreed that they were informed about changes that had been implemented based on the reporting of adverse effects, with no differences between shifts. Finally, 36.4\% (223) disagreed or strongly disagreed with the fact that patient safety was a hospital priority, with rotating staff being more likely to say this $(39.5 \%, 158)$ than day $(33.1 \%, 47)$ or night $(25.4 \%, 17)$ staff $(\mathrm{p}=0.018)$.

When asked about adverse effects, 9.2\% (55) said that patients received the wrong medication several times per month or more: $12.4 \%$ (75) reported the same frequency for pressure ulcers after admission; $1.8 \%$ (11) said there were patient falls in the unit resulting in injuries; $24.2 \%$ (144) said there were nosocomial urinary tract infections; $26.6 \%$ (159) said that there were nosocomial vascular catheter infections; and $20.2 \%$ (120) said that nosocomial pneumonia infections were reported. There was no differences between shifts.

\section{Organisation of provision of care}

We found that $70.8 \%$ (431) of nurses performed non-nursing task sometimes, $59.2 \%$ (361) said they often performed routine blood samples, $52.2 \%$ (317) were sometimes or often responsible for procuring supplies and equipment, and $72.5 \%$ (446) often did administrative tasks and answered the phone. Differences were found between the three shifts in the frequency of transfers and transportation of patients $(\mathrm{p}<0.001)$, in the day shift with night and rotating shifts; for obtaining supplies or equipment $(p=0.001)$ between the day shift and the rotating shift; and answering the phone or performing administrative tasks $(p<0.001)$ between the day and night shifts, and day and rotating shifts (table 2).

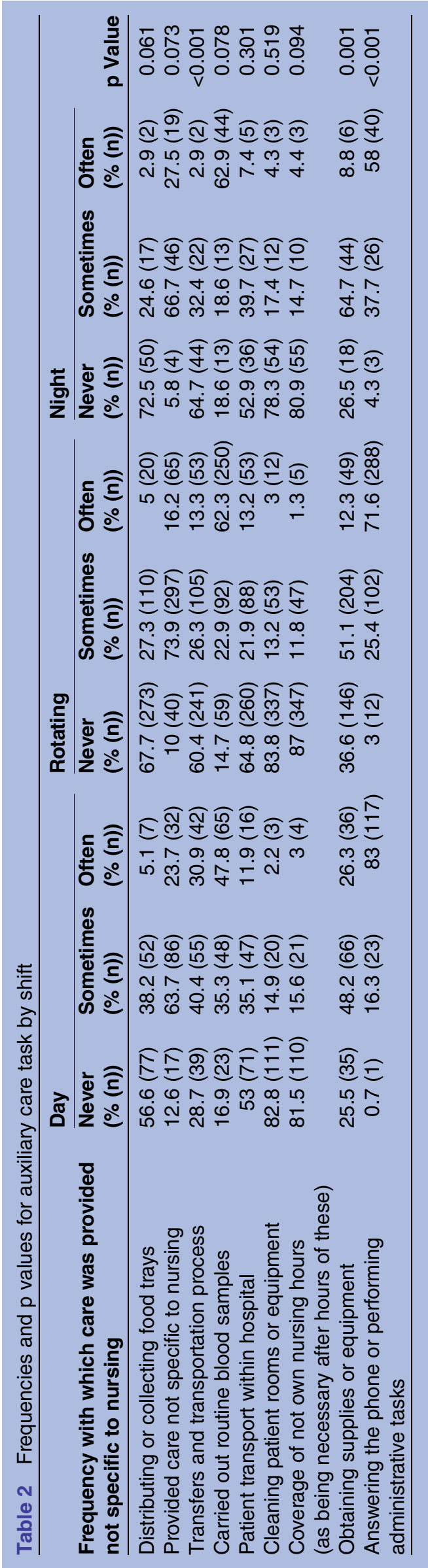


Table 3 Tasks not carried out due to lack of time

\begin{tabular}{|c|c|c|c|c|c|}
\hline \multirow{2}{*}{$\begin{array}{l}\text { During your last day of work, which of the } \\
\text { following activities were necessary but } \\
\text { left due to lack of time? }\end{array}$} & \multirow{2}{*}{$\begin{array}{l}\text { Yes } \\
(n(\%))\end{array}$} & \multicolumn{4}{|c|}{ Actual shift work* } \\
\hline & & $\begin{array}{l}\text { Day } \\
\text { (n (\%)) }\end{array}$ & $\begin{array}{l}\text { Rotating } \\
(\mathrm{n}(\%))\end{array}$ & $\begin{array}{l}\text { Night } \\
\text { (n (\%)) }\end{array}$ & p Value \\
\hline Do or update nursing care plans & $219(34.5)$ & 48 (32.9) & $155(37.8)$ & $15(21.1)$ & 0.021 \\
\hline Health education & $201(31.7)$ & 48 (32.9) & $128(31.2)$ & $23(32.4)$ & 0.927 \\
\hline Oral hygiene & $163(25.7)$ & $36(24.7)$ & $105(25.6)$ & $21(29.6)$ & 0.728 \\
\hline Prepare the patient and his family for discharge & $125(19.7)$ & $27(18.5)$ & $82(20)$ & $16(22.5)$ & 0.782 \\
\hline Speak and confront the patient & $118(18.6)$ & 29 (19.9) & $77(18,8)$ & $12(16.9)$ & 0.871 \\
\hline Plan patient care & $98(15.4)$ & $17(11.6)$ & $70(17.1)$ & $11(15.5)$ & 0.300 \\
\hline Proper registration of nursing care & $81(12.8)$ & $20(13.7)$ & $56(13.7)$ & $4(5.6)$ & 0.161 \\
\hline Skin care & $66(10.4)$ & $10(6.8)$ & $46(11.2)$ & $9(12.7)$ & 0.263 \\
\hline Frequent changing of patient position & $64(10.1)$ & $12(8.2)$ & $45(11)$ & $7(9.9)$ & 0.637 \\
\hline Patient adequate monitoring & $45(7.1)$ & $9(6.2)$ & $30(7.3)$ & $6(8.5)$ & 0.815 \\
\hline Administer medication on time & $35(5.5)$ & $9(6.2)$ & $22(5.4)$ & $4(5.6)$ & 0.937 \\
\hline Treatments and techniques & $7(1.1)$ & $1(0.7)$ & $6(1.5)$ & $0(0)$ & 0.474 \\
\hline Pain management & $4(0.6)$ & $2(1.4)$ & $2(0.5)$ & $0(0)$ & 0.399 \\
\hline
\end{tabular}

*There were eight cases with missing values in the shift work.

Table 4 Levels of burnout

\begin{tabular}{|c|c|c|c|}
\hline & $\begin{array}{l}\text { Emotional } \\
\text { exhaustion } \\
(\%(n))\end{array}$ & $\begin{array}{l}\text { Personal } \\
\text { accomplishment } \\
(\%(n))\end{array}$ & $\begin{array}{l}\text { Depersonalisation } \\
(\%(n))\end{array}$ \\
\hline Low burnout & $57.2(344)$ & $46.4(274)$ & $64.8(388)$ \\
\hline Medium burnout & $25(150)$ & 28.6 (169) & $16.9(101)$ \\
\hline High burnout & $17.8(107)$ & $25(148)$ & $18.4(110)$ \\
\hline $\begin{array}{l}\text { Low general burnout (two or more subscales with } \\
\text { low scores) }\end{array}$ & $58.3(326)$ & & \\
\hline $\begin{array}{l}\text { Medium general burnout (two or more subscales with } \\
\text { medium scores or a different classification in each } \\
\text { subscale) }\end{array}$ & $26.3(147)$ & & \\
\hline $\begin{array}{l}\text { High general burnout (two or more subscales with } \\
\text { high scores) }\end{array}$ & $15.4(86)$ & & \\
\hline
\end{tabular}

Lack of time meant that $18.6 \%$ (118) could not talk to or comfort patients, $31.7 \%$ (201) could not conduct health education, $34.5 \%$ (219) stopped updating nursing care plans and $19.7 \%$ (125) ceased to prepare the patient and family for discharge. There were no differences between shifts apart from a higher frequency of not being able to complete nursing care plans by the rotating shift $(\mathrm{p}=0.021)$ (table 3$)$.

\section{Shift work and nurse outcomes}

With regard to job satisfaction, $76.3 \%$ (473) were moderately or very satisfied with their current job, $60 \%$ (369) were moderately or very satisfied with their professional status and $68.4 \%$ (422) were moderately or very satisfied with the autonomy they had at work, with no differences between shifts. The majority $(85.1 \%, 531)$ were very satisfied with nursing as a career, but this varied between shifts $(p=0.040)$, especially between night and rotating shifts $(\mathrm{p}=0.035$ ), with $15.5 \%$ more staff on rotating shifts being very satisfied $(58.4 \%, 237)$ compared with those on night shifts $(42.9 \%, 30)$. Almost half $(49.4 \%, 307)$ said the work environment was good with $70.4 \%$ (430) saying they would not leave their current position in the next year, with no differences between shifts.

Burnout, measured using the MBI, was low, with $57.2 \%$ (344) reporting emotional exhaustion, $46.4 \%$ (274) reporting personal accomplishment and 64.8\% (388) reporting depersonalisation (table 4). No differences were found between shifts.

\section{Sleep characteristics}

Concerning the assessment of nurses' sleep, 62\% (383) were classified as not definite by the Horne and Östberg Morningness-Eveningness Partial Questionnaire, 17.8\% (110) were moderately morning types and $17 \%$ (105) were moderately evening types. Morningness-eveningness varied between the shifts $(\mathrm{p}=0.004)$, showing a statistically significant difference between the day and rotating shifts $(p=0.006)$ and between the day and night shifts $(p=0.030)$, where day shift staff reflected, in both comparisons, a trend towards the morning type than rotating or night shift staff (table 5). 
Table 5 Cross table between actual shift work and Horne and Östberg Morningness-Eveningness Partial Questionnaire classification

\begin{tabular}{|c|c|c|c|c|c|}
\hline & & \multicolumn{3}{|c|}{$\begin{array}{l}\text { Actual shift work } \\
(\%(n))\end{array}$} & \multirow{2}{*}{$\begin{array}{l}\text { Total } \\
(\%(n))\end{array}$} \\
\hline & & Day & Night & Rotating & \\
\hline \multirow[t]{5}{*}{ H\&ÖP categorisation } & Definite evening type & $2.8(4)$ & $4.3(3)$ & $3(12)$ & $3.1(19)$ \\
\hline & Moderate evening type & $11(16)$ & $18.6(13)$ & $18.9(76)$ & $17(105)$ \\
\hline & Intermediate type & $61.4(89)$ & 60 (42) & $62.5(252)$ & $62(383)$ \\
\hline & Moderate morning type & $24.8(36)$ & $15.7(11)$ & $15.6(63)$ & $17.8(110)$ \\
\hline & Definite morning type & $0(0)$ & $1.4(1)$ & $0(0)$ & $0.2(1)$ \\
\hline
\end{tabular}

H\&ÖP, Horne and Östberg Morningness-Eveningness Partial Questionnaire.

Table 6 Pittsburgh Sleep Quality Index scores (means and differences)

\begin{tabular}{|c|c|c|c|c|c|}
\hline \multirow[b]{2}{*}{ PSQI item } & \multirow[b]{2}{*}{$\begin{array}{l}\text { Global score } \\
\text { Mean (SD) }\end{array}$} & \multicolumn{4}{|c|}{ Actual shift work } \\
\hline & & $\begin{array}{l}\text { Day } \\
\text { Mean (SD) }\end{array}$ & $\begin{array}{l}\text { Rotating } \\
\text { Mean (SD) }\end{array}$ & $\begin{array}{l}\text { Night } \\
\text { Mean (SD) }\end{array}$ & $\begin{array}{l}\text { ANOVA } \\
\text { F ( } p \text { value) }\end{array}$ \\
\hline Item 1: Subjective sleep quality & $1.35(0.641)$ & $1.23(0.624)$ & $1.37(0.619)$ & $1.46(0.774)$ & $3.61(0.028)$ \\
\hline Item 2: Sleep latency & $1.35(0.957)$ & $1.26(0.959)$ & $1.37(0.949)$ & $1.43(1.007)$ & $1.04(0.356)$ \\
\hline Item 3: Sleep duration & $0.76(0.871)$ & $0.92(0.840)$ & $0.66(0.822)$ & $0.97(1.114)$ & $6.82(0.001)$ \\
\hline Item 4: Habitual sleep efficiency & $0.9(1.026)$ & $0.78(0.968)$ & $0.92(1.044)$ & 1.07 (1.033) & $1.68(0.188)$ \\
\hline Item 5: Sleep disturbances & $1.22(0.471)$ & $1.16(0.401)$ & $1.27(0.493)$ & $1.3(0.548)$ & $3.39(0.034)$ \\
\hline Item 6: Use of sleeping medication & $0.38(0.827)$ & $0.36(0.913)$ & $0.35(0.763)$ & $0.6(0.969)$ & $2.87(0.058)$ \\
\hline Item 7: Daytime dysfunction & $0.72(0.726)$ & $0.6(0.669)$ & $0.74(0.715)$ & $0.86(0.879)$ & $3.2(0.041)$ \\
\hline Global score (sum of items) & $6.8(3.387)$ & $6.38(3.427)$ & $6.78(3.261)$ & $7.93(3.804)$ & $4.13(0.017)$ \\
\hline
\end{tabular}

ANOVA F, statistic contrast associated.

PSQI, Pittsburgh Sleep Quality Index.

The Epworth Sleepiness Scale showed that 51.8\% (311) of nurses had excessive daytime sleepiness, while levels were low or absent in $27.7 \%$ (166). No significant differences between shifts were found.

Quality of sleep, measured by the PSQI, averaged 6.8 (SD 3.39) on a scale of 0 (best quality) to 21 (worst quality) (table 6). People working on the night shift had worse quality sleep than those working on the day shift $(p=0.017)$. Table 6 shows the means of the different aspects that define the PSQI, which rates between 0 and 3, with subjective sleep quality and sleep latency having the greatest mean value (1.35) and the use of sleep medication having the lowest of 0.38 (SD 0.83). Significant differences were found between the type of shift and subjective sleep quality score $(\mathrm{p}=0.028)$, sleep duration $(\mathrm{p}=0.001)$, sleep disturbances $(\mathrm{p}=0.034)$ and daytime dysfunction $(p=0.041)$ (table 6). For sleep duration, there were significant differences in sleep duration between rotating and day staff $(\mathrm{p}=0.011)$ and night staff $(p=0.029)$, and a significant difference in sleep disturbances between the day and rotating shifts $(p=0.049)$.

\section{DISCUSSION}

\section{Principal findings}

Nursing requires shift work, and our results reflect the standard distribution of shift patterns in the intensive care, surgical and internal medicine units of hospitals in the SNHS. Rotating shifts with no regular schedule are the most common, with morning or afternoon plus night shifts, and anti-stress shifts of two morning shifts, two afternoon shifts, one night shift and 3 days off. The average shift length is 9 hours, and nurses on night and rotating shifts look after an average of two more patients than day shift nurses. In general, night shift nurses and, sometimes, rotating shift nurses, reported worse perceptions of some organisational and work environmental factors. Similar results have been found ${ }^{40}$ in the limited research literature on this topic. Furthermore, night shift nurses had worse sleep quality, confirming previous evidence. ${ }^{41}$

Only one of four nurses considered the work environment favourable and rated the five PES-NWI subscales lower for positive nurse work environments than the Magnet hospital standards scores. ${ }^{42}$ Our findings identify areas that hospital leaders and policy makers should focus on improving. Nurse participation within nursing and the hospital deserves special attention, as engaging nurses in hospital affairs and reviews of organisational performance has been shown to improve efficiency and effectiveness significantly at the unit level. ${ }^{43}$

Concerning the association between shift work and nurse organisational factors, we found that night shift nurses had worse perceptions of nurse manager ability, leadership and support, suggesting that nurses feel that supervisory staff do not support their practice. This 
result seems logical as hospital activity and the number of nurse managers are reduced at night. Policy makers need to consider this result, because effective supervisors could play a critical role by providing interpersonal and instrumental support, which results in a more supportive and positive team environment. ${ }^{44}$

The nurse work environment is associated with quality and safety of care, ${ }^{15}$ and therefore it makes sense that although the nurses' perception of quality of care and patient safety seemed good, there were an important number of safety items that did not have positive scores. Our findings are in line with the study of Aiken $e t a l^{18}$ carried out in 12 European countries, showing that about a third of nurses considered that patient safety was not a priority and $60 \%$ of nurses disagreed that staff felt free to question the decisions or actions of those in authority. Moreover, night shift nurses in our study were more likely to report that information was lost in the shift change, that errors were used against them and that patient safety was a hospital priority. Rotating and night shift nurses were less confident that patients could manage their self-care after discharge and that the hospital management would act to solve quality and safety problems that they had reported about patient care. Possibly, rotating shift nurses, and especially night shift nurses, perceived a worse quality and safety environment because, as previous research has noted, sleep deprivation affects nurses' abilities to provide the high standard of care they want to give to their patients ${ }^{23}$ and they can find their work more stressful, dangerous and challenging.

In view of earlier results, we were surprised that most nurses estimated that there was a very low frequency of adverse events in their units, including pressure ulcers and injuries from falls, but there was a medium frequency of healthcare associated infections, including nosocomial urinary tract infections and vascular catheter infections. Under reporting of adverse events in healthcare is an acknowledged problem and has been linked to fear of punishment or retribution. ${ }^{5}$ Indeed, in our study, $40 \%$ of nurses agreed that errors were used against them. We did not find an association between shift work and nurses' perceptions about adverse effects, although there has been evidence that performance speed and accuracy during attentional tests are poorer in nurses working night shifts. ${ }^{45}$ In this context, selfreporting is probably not the best instrument to obtain these types of data.

Regarding the organisation of care, approximately one-third of nurses reported having to perform nonnursing tasks often, and similar average percentages were obtained across 12 European countries. ${ }^{46}$ Previous reports have shown large variability between countries, although the most reported tasks ${ }^{47}$ coincide with our findings-namely, answering the phone or performing administrative tasks. An association between non-nursing tasks and lack of time for nursing care has been demonstrated. ${ }^{9}$ The most common nursing care omissions reported by our nurses were developing or updating nursing care plans and conducting health education, the second and third most reported activities left undone in the 488 European hospitals from 12 countries. ${ }^{9}$ Our findings also confirm the results of the European study ${ }^{31}$ where activities reflecting physical care and monitoring were less frequently omitted. We found day shift nurses were more likely to carry out nonnursing activities, such as transfers and transport, obtaining supplies or equipment, answering the phone or performing administrative tasks. This is not surprising considering that planned activities are concentrated during the day shift, such as scheduled admissions and routine tests.

With regard to nurse outcomes, most Spanish nurses stated they were satisfied with their job, in line with the average percentages found in 12 European countries. ${ }^{19}$ Moreover, $60 \%$ of nurses confirmed being satisfied with the professional status and autonomy they had at work. All Spanish nurses have a bachelor degree, and previous research showed that nurses with degrees reported higher job satisfaction. ${ }^{27}$ Concerning the relation between shift work and job satisfaction, our results did not show any significant differences, with only $15 \%$ more of rotating shift nurses being more satisfied with their profession than night shift nurses.

A meta-analysis ${ }^{48}$ showed a high and significant correlation between nurses' job satisfaction and burnout. A high percentage of nurses in our study were satisfied with their job; this could explain the low level of nurses with high general burnout in our study. It is difficult to compare our results with previous research because of the variability in cut-off points, but our findings were very moderate compared with others. ${ }^{15}{ }^{49}$ In contrast, previous studies showed a relationship between healthcare shift work and some of the three dimensions of burnout. ${ }^{26}$ However, the above mentioned meta-analysis study $^{48}$ did not find a correlation between shift work and any of the three MBI dimensions. It is difficult to explain the differences found; variations in health systems and health organisations could explain some of the differences in the results. For example, the evidence suggests that appropriate sleeping hours and adequate days off are possible protective factors, ${ }^{26}$ but higher nurse/patient ratios have been linked to burnout. ${ }^{12}$

Finally, our nurses showed similar scores in global sleep quality to a previous study, ${ }^{50}$ but slightly higher ${ }^{51}$ and lower $^{52}$ than others. Differences in global sleep quality scores between day and night shift staff were found, echoing previous research. ${ }^{45}$ Our findings also suggest that night shift nurses had worse subjective sleep quality, daytime dysfunction and sleep disturbances than day shift nurses. This could be because night shifts disturb circadian rhythms and induce less robust activity rhythms. ${ }^{53}$ Encouraging shift workers to sleep longer on their first day after starting night duty is therefore recommended. ${ }^{45}$

However, we must interpret the results carefully. In line with the literature, ${ }^{23}$ we found that a higher percentage 
of day shift nurses are morning types compared with staff working on a rotating or night shift, and the evidence ${ }^{54}$ indicates that evening type nurses have a significantly increased risk of worse sleep quality.

Rotating shift nurses obtained better results for sleep duration than day and night shift staff. Chung et $a \bar{l}^{5}$ suggested that rotating shift nurses may know that shift patterns induce further irregular sleep-wake times so they usually try to sleep longer at night.

Half of the nurses reported excessive sleepiness, which was higher than in other studies. ${ }^{56}$ We did not find any differences in the sleepiness of nurses working on different shifts, in contrast with studies conducted with different workers, ${ }^{21} 2945$ but similar to Norwegian nurses. ${ }^{57}$ Nevertheless, managers and administrators should consider these findings as important, as sleepiness is associated with an increased likelihood of errors. $^{58}$

\section{Conclusions about shift schedules}

The only significant difference that we found related to the work environment was in the PES factor 'nurse manager ability, leadership and support', with night shift workers having a worse work environment than day staff. The other differences were not significant.

Night shift nurses were more likely to report that relevant information was lost during shift changes, and rotating and night shift nurses were less confident about patient self-care competence after discharge. Although the nurses' perceptions of quality of care and patient safety seemed good, we found a significant number of adverse effects. The most common nursing care omissions were related to nursing care plans, with one-third of nurses reporting they often performed non-nursing tasks and they had little time for patient health education.

Our results did not show any differences regarding the relationship between shift work and job satisfaction, and only one in six rotating shift nurses were more satisfied that they chose nursing as a profession than night shift nurses.

There was a difference between the global sleep quality score for day and night shift nurses. Day nurses reported better sleep quality, while rotating shift nurses obtained better scores for sleep duration than the other two groups.

\section{Potential limitations}

Our study had a number of strengths-for example, 635 nurses from seven Spanish hospitals took part in the study, representing hospitals of different sizes (small, medium and large) and different specialties (surgical, medical and critical care). Our study also had some limitations. First, the cross sectional design limited our ability to infer causal relationships between the characteristics of nurses' work environment in hospitals in the SNHS, nurse reported quality of care and the organisation of the provision of care through different shifts schemes. Second, the survey did not include some aspects of shift work, including the number of hours of overtime, mode of overtime, the possibility of taking breaks during shifts and total hours worked per week. Hence it is possible that some unmeasured factors were not included. Furthermore, we were not able to include any information about nurses' work-life balance, or about the proportion of nurses with family commitments.

\section{Conclusions and policy implications}

This study provides data about work environment, quality and safety, and organisation of provision of care collected when austerity measures were leading to cuts in spending on public services. Only 20\% (95) of Spanish nurses considered that their work environment was favourable, and this study helps to identify precise areas that should be improved. This is one of the first studies that has been primarily designed to investigate shift work and the relationship with nurse organisational factors and nurse reported quality of care. It provides evidence for nurses, managers and policy makers on the impact of shift work, to inform decisions on nurse working patterns and guarantee the welfare of nurses and the quality of care that patients receive.

\section{Author affiliations}

${ }^{1}$ Nursing and Healthcare Research Unit (Investén-isciii), Institute of Health Carlos III, Ministry of Science and Innovation, Madrid, Spain

${ }^{2}$ Faculty of Nursing, University of Murcia, Murcia, Spain

${ }^{3}$ Vall d'Hebron Hospital, Nursing and Healthcare Research, Barcelona, Spain

${ }^{4}$ IMIB-Arrixaca, CronoLab, Department of Physiology, University of Murcia, Murcia, Spain

${ }^{5}$ Department of Physiology, University of Murcia, Murcia, Spain

Collaborators The following are members of the SYCE and RETICEF group: Emma Alonso Poncelas, Beatriz Baños Otalora, M ${ }^{a}$ de los Ángeles Bonmati, Silvia Esteban Sepúlveda, Carmen Fuentelsaz Gallego, Berta García Fraguela, Teresa Gómez-García, Esther González-María, Mª del Pilar Heredia Reina, Mª Lidón López Iborra, Alejandro Lúcas Sánchez, Juan Antonio Madrid Pérez, $\mathrm{M}^{\mathrm{a}}$ José Martínez Madrid, Antonio Martínez Nicolás, Teresa Moreno-Casbas, Elisabeth Ortiz Tudela, $M^{\circ}$ Pilar Pérez Sánchez, Maria Ángeles Rol de Lama and María Ruzafa-Martínez.

Contributors TG-G designed the nurses' survey, coordinated the data collection, wrote the statistical analysis plan, cleaned and analysed the data, and drafted and revised the paper. MR-M initiated the project, designed the nurses' survey, coordinated the data collection, wrote the statistical analysis plan, and drafted and revised the paper. CF-G initiated the project, the nurses' survey, coordinated the data collection, wrote the statistical analysis plan, and drafted and revised the paper. JAM coordinated the data collection, and drafted and revised the paper. MAR coordinated the data collection, and drafted and revised the paper. MJM-M coordinated the data collection, and drafted and revised the paper. TM-C initiated the project, designed the nurses' survey, coordinated the data collection, wrote the statistical analysis plan, cleaned and analysed the data, and drafted and revised the paper. She is the guarantor.

Funding This study was carried out as part of a project entitled 'Functioning of the circadian system, working environment, and the organisation of nursing care of hospitals of the National Health System', financed by the Spanish Health Research Fund (PI 11/00646, Health Ministry), the Ministry of Science and Innovation (SAF2013-49132-C2-1-R) and the Institute of Health Carlos III (RETICEF, RD12/0043/0011, RD12/0043/0006).

Competing interests All authors had financial support from the Spanish Health Research Fund, the Ministry of Science and Innovation, and from the Institute of Heath Carlos III for the submitted work. 
Ethics approval The project was approved by the Spanish Health Research Fund (Fondo de Investigaciones Sanitarias PI11/00646).

Provenance and peer review Not commissioned; externally peer reviewed.

Data sharing statement No additional data are available.

Open Access This is an Open Access article distributed in accordance with the Creative Commons Attribution Non Commercial (CC BY-NC 4.0) license, which permits others to distribute, remix, adapt, build upon this work noncommercially, and license their derivative works on different terms, provided the original work is properly cited and the use is non-commercial. See: http:// creativecommons.org/licenses/by-nc/4.0/

\section{REFERENCES}

1. International Council of Nurses, International Pharmaceutica Federation, International Hospital Federation, World Confederation for Physical Therapy, World Dental Federation, World Medical Association. Positive practice environments for health care professionals. 2008. http://www.whpa.org/PPE_Fact_Health_Pro.pdf (accessed 8 Feb 2016).

2. WHO. European strategic directions for strengthening nursing and midwifery towards Health 2020 goals. Marmorvej, 2015. http://www. euro.who.int/_data/assets/pdf_file/0004/274306/Europeanstrategic-directions-strengthening-nursing-midwifery-Health2020_ en-REV1.pdf?ua=1 (accessed 7 Feb 2016)

3. Corner DJ, Glasper A. Does UK nursing research have an impact on care delivery? Br J Nurs 2015;24:294-5.

4. Aiken LH, Sloane DM, Clarke S, et al. Importance of work environments on hospital outcomes in nine countries. Int J Qual Health Care 2011;23:357-64.

5. Kirwan M, Matthews A, Scott PA. The impact of the work environment of nurses on patient safety outcomes: a multi-level modelling approach. Int J Nurs Stud 2013;50:253-63.

6. Stimpfel AW, Sloane DM, McHugh MD, et al. Changes in patient and nurse outcomes associated with magnet hospital recognition. Int J Nurs Stud 2013;50:126-35.

7. Lee $\mathrm{SY}$, Kim CW, Kang JH, et al. Influence of the nursing practice environment on job satisfaction and turnover intention. J Prev Med Public Health 2014;47:258-65.

8. Cho E, Sloane DM, Kim EY, et al. Effects of nurse staffing, work environments, and education on patient mortality: an observational study. Int J Nurs Stud 2015;52:535-42.

9. Ausserhofer D, Zander B, Busse R, et al. Prevalence, patterns and predictors of nursing care left undone in European hospitals: results from the multicountry cross-sectional RN4CAST study. BMJ Qual Saf 2014:23:126-35

10. Ma C, Park SH. Hospital Magnet status, unit work environment, and pressure ulcers. J Nurs Scholarsh 2015;47:565-73.

11. Ma C, McHugh MD, Aiken LH. Organization of hospital nursing and 30-day readmissions in medicare patients undergoing surgery. Med Care 2015;53:65-70.

12. Kutney-Lee A, Stimpfel AW, Sloane DM, et al. Changes in patient and nurse outcomes associated with magnet hospital recognition. Med Care 2015;53:550-7.

13. Stimpfel AW, Sloane DM, McHugh MD, et al. Hospitals known for nursing excellence associated with better hospital experience for patients. Health Serv Res 2016;51:1120-34.

14. Burton J. WHO healthy workplace framework and model: background and supporting literature and practices. Geneva Switzerland, 2010. http://www.whpa.org/PPE_Fact_Health_Pro.pdf (accessed 8 Feb 2016)

15. Aiken LH, Sermeus W, Van den Heede K, et al. Patient safety, satisfaction, and quality of hospital care: cross sectional surveys of nurses and patients in 12 countries in Europe and the United States. BMJ 2012;344:e1717.

16. Lacher S, De Geest S, Denhaerynck K, et al. The quality of nurses' work environment and workforce outcomes from the perspective of Swiss allied healthcare assistants and registered nurses: a cross-sectional survey. J Nurs Scholarsh 2015;47:458-67.

17. Li B, Bruyneel L, Sermeus W, et al. Group-level impact of work environment dimensions on burnout experiences among nurses: a multivariate multilevel probit model. Int J Nurs Stud 2013;50:281-91.

18. Aiken LH, Sloane DM, Bruyneel L, et al. Nurses' reports of working conditions and hospital quality of care in 12 countries in Europe. Int J Nurs Stud 2013;50:143-53.

19. Dall'Ora C, Griffiths P, Ball J, et al. Association of $12 \mathrm{~h}$ shifts and nurses' job satisfaction, burnout and intention to leave: findings from a cross-sectional study of 12 European countries. BMJ Open 2015;5:e008331.

20. Harris R, Sims S, Parr J, et al. Impact of $12 \mathrm{~h}$ shift patterns in nursing: a scoping review. Int J Nurs Stud 2015;52:605-34.

21. Boivin DB, Boudreau P. Impacts of shift work on sleep and circadian rhythms. Pathol Biol 2014;62:292-301.

22. Boissard $\mathrm{P}$, Cartron $\mathrm{D}$, Gollac $\mathrm{M}$, et al. Time and work: duration of work. Dublin, Ireland: European Foundation for the Improvement of Living and Working Conditions, 2003. ISBN 92-897-0154-4.

23. Mueckes S. Effects of rotating night shifts: literature review. J Adv Nurs 2005;50:433-9.

24. Ramoo V, Abdullah KL, Piaw CY. The relationship between job satisfaction and intention to leave current employment among registered nurses in a teaching hospital. J Clin Nurs 2013;22:3141-52.

25. Stimpfel AW, Sloane DM, Aiken LH. The longer the shifts for hospita nurses, the higher the levels of burnout and patient dissatisfaction. Health Aff (Millwood) 2012;31:2501-9.

26. Wisetborisut A, Angkurawaranon C, Jiraporncharoen W, et al. Shift work and burnout among health care workers. Occup Med (Lond) 2014;64:279-86.

27. Korompeli A, Muurlink O, Tzavara C, et al. Influence of shiftwork on Greek nursing personnel. Saf Health Work 2014;5:73-9.

28. Killgore WDS, Grugle NL, Balkin TJ. Gambling when sleep deprived don't bet on stimulants. Chronobiol Int 2012;29:43-54.

29. Caruso CC. Negative impacts of shiftwork and long work hours. Rehabil Nurs 2014;39:16-25.

30. Van Dongen HPA, Belenky G. Individual differences in vulnerability to sleep loss in the work environment. Ind Health 2009;47:518-26.

31. Sermeus W, Aiken LH, Van den Heede K, et al. Nurse forecasting in Europe (RN4CAST): rationale, design and methodology. BMC Nurs 2011;10:6

32. Fuentelsaz-Gallego C, Moreno-Casbas MT, Gonzalez-Maria E. Validation of the Spanish version of the questionnaire Practice Environment Scale of the Nursing Work Index. Int J Nurs Stud 2013;50:274-80.

33. Seisdedos N. MBI. Inventario Burnout de Maslach: manual (MBI, the Maslach Burnout Inventory Manual). Madrid: TEA Ediciones, S.A, 1997.

34. Maslach C, Jackson SE. The Maslach burnout inventory. 2nd edn. Pal Alto, California: Consulting Psychologists Press, 1986:20.

35. Fuentelsaz-Gallego C, Moreno-Casbas T, López-Zorraquino D, et al. en nombre del consorcio RN4CAST-España. Percepción del entorno laboral de las enfermeras españolas en los hospitales del Sistema Nacional de Salud. Proyecto RN4CAST-España. Enferm Clin 2012;22:261-8.

36. Honer J, Ostberg O. Self-assessment questionnaire to determine morningness-eveningness in human circadian rhythms. Int J Chronobiol 1976;4:97-110.

37. Johns MW. A new methods for measuring daytime sleepiness: the Epworth Sleepiness Scale. Sleep 1991;14:540-5.

38. Buysse DJ, Reinolds CF, Monk TH, et al. The Pittsburg Sleep Quality Index: a new instrument for psychiatric practice and research. Psychiatry Res 1989;28:193-213.

39. Moreno-Casbas MT, Ruzafa-Martinez M, Rol MA, et al. SYCE Network. Sleepiness in Spanish nursing staff-influence of chronotype and care unit in circadian rhythm impairment: research protocol. J Adv Nurs 2014;70:211-19.

40. Teclaw R, Osatuke K. Nurse perceptions of workplace environment: differences across shifts. J Nurs Manag 2015;23: 1137-46.

41. Lin SH, Liao WC, Chen MY, et al. The impact of shift work on nurses' job stress, sleep quality and self-perceived health status. J Nurs Manag 2014;22:604-12.

42. Desmedt M, De Geest S, Schubert M, et al. A multi-method study on the quality of the nurse work environment in acute-care hospitals: positioning Switzerland in the Magnet hospital research. Swiss Med Wkly 2012;142:w13733

43. Drenkard K. The business case for Magnet. J Nurs Adm 2010;40:263-71.

44. Pisarski A, Lawrence SA, Bohle $P$, et al. Organizational influences on the work life conflict and health of shiftworkers. Appl Ergon. 2008;39:580-8.

45. Niu SF, Chu $\mathrm{H}$, Chen $\mathrm{CH}$, et al. A comparison of the effects of fixedand rotating-shift schedules on nursing staff attention levels a randomized trial. Biol Res Nurs 2013;15:443-50.

46. Bruyneel L, Li B, Ausserhofer D, et al. Organization of hospital nursing, provision of nursing care, and patient experiences with care in Europe. Med Care Res Rev 2015;72:643-64.

47. Bruyneel L, Li B, Aiken L, et al. A multi-country perspective on nurses' tasks below their skill level: reports from domestically trained 
nurses and foreign trained nurses from developing countries. Int J Nurs Stud 2013;50:202-9.

48. Vargas C, Cañadas GA, Aguayo R, et al. Which occupational risk factors are associated with burnout in nursing? A meta-analytic study. Int J Clin Heal Psychol 2014;14:28-38.

49. Cañadas-De la Fuente GA, Vargas C, San Luis C, et al. Risk factors and prevalence of burnout syndrome in the nursing profession. Int J Nurs Stud 2015;52:240-9.

50. Arimura M, Imai M, Okawa M, et al. Sleep, mental health status, and medical errors among hospital nurses in Japan. Ind Health 2010;48:811-17.

51. Shao MF, Chou YC, Yeh MY, et al. Sleep quality and quality of life in female shift-working nurses. J Adv Nurs 2010;66:1565-72.

52. Karagozoglu S, Bingol N. Sleep quality and job satisfaction of Turkish nurses. Nurs Outlook 2008;56:298-307.e3.

53. Kang JH, Miao NF, Tseng IJ, et al. Circadian activity rhythms and sleep in nurses working fixed 8-hr shifts. Biol Res Nurs $2015 ; 17: 348-55$
54. Chung MH, Chang FM, Yang $\mathrm{CCH}$, et al. Sleep quality and morningness-eveningness of shift nurses. J Clin Nurs 2009;18:279-84.

55. Chung MH, Liu WI, Lee HL, et al. Selected neurophysiological, psychological, and behavioral influences on subjective sleep quality in nurses: a structure equation model. PLOS ONE 2013;8: e79529.

56. Eldevik MF, Flo E, Moen BE, et al. Insomnia, excessive sleepiness, excessive fatigue, anxiety, depression and shift work disorder in nurses having less than 11 hours in-between shifts. PLOS ONE 2013;8:e70882.

57. Øyane NMF, Pallesen S, Moen BE, et al. Associations between night work and anxiety, depression, insomnia, sleepiness and fatigue in a sample of Norwegian nurses. PLOS ONE 2013;8: e70228.

58. Dorrian J, Lamond N, van den Heuvel C, et al. A pilot study of the safety implications of Australian nurses' sleep and work hours. Chronobiol Int 2006;23:1149-63. 